Check for updates

Cite this: RSC Adv., 2019, 9, 22540

Received 5th December 2018

Accepted 26th June 2019

DOI: $10.1039 / c 8 r a 09994 d$

rsc.li/rsc-advances

\section{Optimization of the extraction, preliminary characterization, and anti-inflammatory activity of crude polysaccharides from the stems of Trapa quadrispinosa}

\begin{abstract}
Feng Li, ${ }^{a c}$ Xinhu Liu, ${ }^{b}$ Xiaofeng Yu, ${ }^{c}$ Xiuquan Xu (DD *c and Huan Yang*c
A novel method was developed using pressurized-assisted extraction (PAE) to efficiently extract crude Trapa quadrispinosa polysaccharides (TQCPS) from plant stems, and the extraction process was optimized using response surface methodology (RSM). At a water-to-material fixed ratio of $30 \mathrm{~mL} \mathrm{~g}^{-1}$, the highest yield of $3.72 \pm 0.13 \%$ was obtained under the optimum conditions of extraction time of $32 \mathrm{~min}$, extraction temperature at $47^{\circ} \mathrm{C}$, and extraction pressure at $1.87 \mathrm{Mpa}$, which were in agreement with the predicted value of $3.683 \%$. Compared with conventional hot water extraction (HWE), the PAE method remarkably enhanced the extraction yield with the further advantages of short extraction time and low extraction temperature. The preliminary characteristics of TQCPS were analyzed through UVvis, FT-IR, and chemical composition analysis. In subsequent anti-inflammatory studies, when RAW 264.7 mouse macrophage cells were treated with TQCPS, satisfactory anti-inflammatory activity was observed, and TQCPS significantly suppressed the release of nitric oxide (NO), tumor necrosis factor- $\alpha$ (TNF- $\alpha$ ), and interleukin-6 (IL-6) and synchronously restrained the expression levels of inducible nitric oxide synthase (iNOS), TNF- $\alpha$, and IL- 6 mRNA induced by lipopolysaccharide (LPS) in a dose-dependent manner. These results indicate that PAE is a technology that can be used for efficient extraction of polysaccharides from medicinal plants, and TQCPS can be explored as a potential anti-inflammatory agent in medicine.
\end{abstract}

\section{Introduction}

Polysaccharides are a type of essential primary metabolite that exists in animals, plants, and fungi. In recent decades, there has been great interest in polysaccharides isolated from plants due to their various biological activities including anti-oxidant, antimicrobial, anti-tumor, anti-diabetic, and anti-inflammatory activities. $^{1-5}$ Inflammation is a complicated biological response against injury, infection, and stress, and is mainly mediated by pro-inflammatory cells including macrophages. ${ }^{6}$ During the process of inflammation, activated macrophages can synthesize and release various inflammatory cytokines including tumor necrosis factor (TNF)- $\alpha$, interleukin (IL)-1 $\beta$, IL6 , and nitric oxide (NO) to promote inflammation or fight against pathogens. ${ }^{7}$ However, excess or inappropriate production of these inflammatory cytokines can also cause various inflammatory diseases such as rheumatoid arthritis, osteoarthritis, diabetes, Alzheimer's disease, and even cancer. ${ }^{8}$

${ }^{a}$ Affiliated Hospital of Jiangsu University, Zhenjiang 212001, P. R. China ${ }^{b}$ Taizhou National Medical Hitech Development Zone, Taizhou 225300, P. R. China ${ }^{c}$ School of Pharmacy, Jiangsu University, Zhenjiang 212013, P. R. China. E-mail: xxq781026@ujs.edu.cn; Huanyang198109@163.com
Therefore, the inhibition of the synthesis or release of these inflammatory cytokines is an important target for the treatment of inflammatory diseases. It has been demonstrated that some natural polysaccharides exhibit obvious anti-inflammatory activities by inhibiting the production of inflammatory cytokines in cell or mouse models., ${ }^{9,10}$

It is important to be able to efficiently extract these bioactive polysaccharides for their application or further research. Conventionally, polysaccharides are extracted from plant tissues by heating, boiling, or refluxing methods. However, these techniques have many disadvantages including requiring a great deal of time and energy, low yields, and degradation of polysaccharides with the loss of their pharmacological activities. ${ }^{11}$ It is necessary to find an efficient method for extracting polysaccharides in order to avoid these disadvantages of traditional extraction techniques. Recently, various advanced techniques have been developed to extract bioactive polysaccharides, such as ultrasonic-assisted extraction (UAE), microwave-assisted extraction (MAE), enzyme-assisted extraction (EAE), and pressurized-assisted extraction (PAE). ${ }^{12-15}$ Among these techniques, PAE is considered to be a relatively innovative and promising technique due to its higher efficiency and environmentally friendly characteristics of lower energy 
consumption and reduced emission of pollutant solvents. ${ }^{16,17}$ In the PAE process, the higher temperature and pressure increases the solubility and diffusion rate of target compounds, and reduces the viscosity and surface tension of the solvent, allowing increased penetration into the samples and thus achieving efficient extraction and remarkable activities over a wide range of polarities of compounds. ${ }^{18-21}$ PWE has been efficiently applied for extracting bioactive substances such as phenolic compounds and polysaccharide from different plants. ${ }^{22-24}$ Usually, extraction of bioactive substances from plant tissues by PAE is influenced by multiple independent variables such as extraction temperature, extraction time, extraction pressure, particle size, and liquid-to-sample ratio and their mutual interactions. Response surface methodology (RSM), an effective statistical technique, can be used to investigate and optimize complex processes when the independent variables have a combined effect on the response variables. ${ }^{25}$ The main advantage of RSM is to significantly reduce the experimental trials that are required for evaluating multiple parameters and their interactions. ${ }^{26}$

Trapa quadrispinosa, also named as Sijiaoling and belonging to the Trapaceae family, is regarded as a popular vegetable for its wonderful flavor and medicinal functions. ${ }^{27}$ The pericarps of T. quadrispinosa are used in allaying fever, diffusing dampness, dispelling pathogenic wind, and invigorating the spleen in traditional Chinese medicine. ${ }^{28}$ However, to the best of our knowledge, the composition and biological activities of the stem of T. quadrispinosa have rarely been examined.

In our previous study, polysaccharides were detected in the stem of T. quadrispinosa, and they engaged in a variety of free radical scavenging activities. ${ }^{29}$ It is well known that reactive oxygen species (ROS) are closely related to the occurrence and development of inflammation and tumors. ${ }^{30}$ Trapa quadrispinosa polysaccharides (TQCPS) possess excellent antioxidant abilities, and may exhibit satisfactory anti-inflammatory activity. However, there is very little data on the extraction and anti-inflammatory activity of TQCPS. Thus, the objective of the present study was to explore the potential use of pressure technology on the extraction of crude polysaccharides from the stem of T. quadrispinosa and evaluate their anti-inflammatory activity.

\section{Experimental}

\subsection{Reagents and materials}

The stems of T. quadrispinosa were collected from Weishan Lake, Weishan County, Shandong province, China. The dried materials were pulverized and screened through a 60-mesh sieve. The powder was extracted with petroleum ether $\left(60-90^{\circ} \mathrm{C}\right)$ and $95 \%$ ethanol for $6 \mathrm{~h}$ in a Soxhlet apparatus to remove some colored materials and other micromolecular substances. Thereafter, the samples were obtained by filtration and dried for further use.

The RAW 264.7 murine macrophage cell line was obtained from the American Type Culture Collection (Rockville, MD, USA). Lipopolysaccharide (LPS) from Escherichia coli (0111:B4), 3-(4,5-dimethyl-2-thiazolyl)-2,5-diphenyl- $2 H_{-}$ tetrazolium bromide (MTT), and Griess reagent were purchased from Sigma-Aldrich Co. (St. Louis, MO, USA). Dulbecco's modified Eagle's medium (DMEM), fetal bovine serum (FBS), streptomycin, penicillin, and TRIzol reagent were all purchased from Gibco Laboratories (Grand Island, NY, USA). Mouse IL-6 and TNF- $\alpha$ enzyme-linked immunosorbent assay (ELISA) kits were purchased from Invitrogen (Carlsbad CA, USA). All other chemical reagents used in experiments were of analytical grade, and double-distilled water was used throughout the experiments.

\subsection{Pressure-assisted extraction (PAE)}

The PAE was carried out in a high pressure micro-reactor (WHF$0.25 \mathrm{~L}$, automatically controlled reaction kettle, Weihai Co. Ltd., Shandong, China). Samples ( $2.0 \mathrm{~g})$ were extracted with distilled water at different temperatures $\left(30-70{ }^{\circ} \mathrm{C}\right)$ and different waterto-material ratios $\left(20-40 \mathrm{~mL} \mathrm{~g}^{-1}\right)$ under extraction pressure (1.2-2.4 Mpa) for various periods (10-50 min). After extraction, the mixtures were centrifuged at $4000 \mathrm{rpm}$ for $10 \mathrm{~min}$, and the collected supernatants were concentrated to a certain volume by a rotary evaporator. Then, the crude polysaccharides were precipitated by the addition of absolute ethanol to a final concentration of $80 \%(\mathrm{v} / \mathrm{v})$ at $4{ }^{\circ} \mathrm{C}$ for $24 \mathrm{~h}$. The obtained crude polysaccharides were re-dissolved in double-distilled water for polysaccharide content and anti-inflammatory activity determination.

The percentage yield of polysaccharide from $T$. quadrispinosa stems was determined with the phenol-sulfuric method. ${ }^{31}$ Glucose was chosen as a standard, and the polysaccharide yield (\%) was calculated as follows:

$$
\begin{aligned}
& \text { Polysaccharide yield }(/ \%)= \\
& \qquad \frac{\text { polysaccaride content of extracts }(\mathrm{g})}{\text { weight of sample powder }(\mathrm{g})} \times 100
\end{aligned}
$$

For comparison, in hot water extraction (HWE), a $5.0 \mathrm{~g}$ sample was extracted using $150 \mathrm{~mL}$ of distilled water at $80^{\circ} \mathrm{C}$ for $4 \mathrm{~h}$.

Based on the preliminary single factor results, RSM was employed to further optimize the PAE conditions for TQCPS from the stems of T. quadrispinosa. Extraction time $\left(X_{1}, \mathrm{~min}\right)$, extraction temperature $\left(X_{2},{ }^{\circ} \mathrm{C}\right)$, and extraction pressure $\left(X_{3}\right.$, $\mathrm{Mpa}$ ) were the preferred independent variables. A Box-Behnken Design (BBD) with three variables and three levels was constructed. The yield of TQCPS was designated as the responsedependent value. Based on the experimental data from the BBD, regression analysis was carried out, and a second-order polynomial model was obtained as follows:

$$
Y=A_{0}+\sum_{i=1}^{3} A_{i} X_{i}+\sum_{i=1}^{3} A_{i i} X_{i}^{2}+\sum_{i=1}^{2} \sum_{j=i+1}^{3} A_{i j} X_{i} X_{j}
$$

where $Y$ denotes the predicted response; $A_{0}$ denotes a constant, $A_{i}$ denotes a linear coefficient, $A_{i i}$ denotes a quadratic coefficient, $A_{i i}$ denotes an interaction coefficient, and $X_{i}$ and $X_{j}$ denote independent variables. 
Design-Expert version 8.0.5b software (Stat-Ease Inc., Minneapolis, MN, USA) was used to analyze the experimental data. Analysis of variance (ANOVA) was used to evaluate the significant terms in the model. The various statistical analysis parameters including lack of fit tests, $P$ value, $F$ value, determination coefficient $\left(R^{2}\right)$, adjusted determination coefficient $\left(R_{\mathrm{adj}}{ }^{2}\right)$, and coefficient of variation (C.V.) were chosen to evaluate the adequacy of the models.

\subsection{Scanning electron microscopy analysis}

In order to understand the mechanism of PAE, the microstructure of an untreated sample as well as residue after extraction were collected and observed by scanning electron microscopy (SEM). The dried sample powders were fixed on a specific carbon film support and covered with gold using a sputter coater. The shape and the surface characteristics were observed by a SEM system (Hitachi S4800, Japan) under high vacuum conditions.

\subsection{Analysis of TQCPS}

2.4.1. Chemical composition analysis of TQCPS. The carbohydrate content in TQCPS was determined through the above-mentioned phenol-sulfuric acid method. The uronic acid content was measured according to the method of Blumenkrantz and Asboe-Hansen, with galacturonic acid as the standard. ${ }^{32}$ The protein content was determined by the Bradford method using bovine serum albumin (BSA) as the standard. ${ }^{33}$ The total polyphenol content was evaluated by Folin-Ciocalteu assay with gallic acid as the standard. ${ }^{34}$

2.4.2. Ultraviolet (UV) and Fourier transform infrared (FTIR) spectroscopic analysis of TQCPS. The UV spectrum of TQCPS was recorded on a UV-vis spectrophotometer (Shimadzu UV-2600, Japan) from $200 \mathrm{~nm}$ to $600 \mathrm{~nm}$ with distilled water as the blank. The FT-IR spectrum of TQCPS was identified by a Fourier transform infrared spectrophotometer (Avatar 370, Nicolet Co., USA). The sample was evenly mixed with dried $\mathrm{KBr}$ powder and pressed into pellets for FT-IR determination over the wavelength range of $4000-400 \mathrm{~cm}^{-1}$.

\subsection{Anti-inflammatory activity assay in vitro}

The anti-inflammatory effects of TQCPS were evaluated using the LPS-induced RAW 264.7 cell assay as previously reported. ${ }^{7}$

2.5.1. Cell culture. Murine macrophage RAW 264.7 cells were cultured in DMEM medium supplemented with $10 \% \mathrm{FBS}$, $100 \mathrm{U} \mathrm{mL}^{-1}$ penicillin, and $100 \mu \mathrm{g} \mathrm{mL}{ }^{-1}$ streptomycin at $37^{\circ} \mathrm{C}$ in a humidified atmosphere with $5 \% \mathrm{CO}_{2}$. Cells in log phase were used for experiments.

2.5.2. Cell viability assay. The viability of RAW 264.7 cells was determined by MTT colorimetric assay. Briefly, RAW 264.7 cells were seeded into 96-well plates at an optimum density of 2 $\times 10^{4}$ cells per well and incubated for $12 \mathrm{~h}$, and then treated with different TQCPS concentrations for different time periods. Following treatment, $10 \mu \mathrm{L}$ of MTT $\left(5 \mathrm{mg} \mathrm{mL}^{-1}\right)$ was added to each well, and the plates were further incubated for $4 \mathrm{~h}$. After adding $150 \mu \mathrm{L}$ of DMSO to dissolve formazan crystals, the absorbance of each well was measured at $570 \mathrm{~nm}$ using an ELISA reader.

2.5.3. Nitric oxide and cytokine assays. The concentration of nitric oxide in the culture supernatants was measured by Griess reaction. The RAW 264.7 cells at a density of $2 \times 10^{4}$ cells per well were pre-incubated with various concentrations of TQCPS for $1 \mathrm{~h}$, and then stimulated by LPS (final concentration $0.5 \mu \mathrm{g} \mathrm{mL}{ }^{-1}$ ) for $24 \mathrm{~h}$ at $37{ }^{\circ} \mathrm{C}$. After stimulation, $100 \mu \mathrm{L}$ supernatant was mixed with $100 \mu \mathrm{L}$ of Griess reagent, and the solution was incubated at room temperature for $10 \mathrm{~min}$. Finally, the absorbance of the mixture was determined at $540 \mathrm{~nm}$. The level of nitric oxide was measured based on the standard curves established with serial dilutions of $\mathrm{NaNO}_{2}$.

For the cytokine assay, after stimulation by LPS, the cell culture supernatants were collected, and the concentrations of IL-6 and TNF- $\alpha$ were carefully determined using ELISA kits according to the manufacturer's instructions.

2.5.4. Quantitative real-time PCR assay. The RAW 264.7 cells were treated with various concentrations of TQCPS in the absence of LPS $\left(0.5 \mu \mathrm{g} \mathrm{mL}{ }^{-1}\right)$ for $12 \mathrm{~h}$. Subsequently, the cells were separated from the medium by centrifugation, and the total RNA was isolated using TRIzol reagent according to the manufacturer's instructions. Then, $1.0 \mu \mathrm{g}$ total RNA was reverse transcribed to produce cDNAs using a RevertAid First Strand cDNA Synthesis Kit. RT-PCR was carried out using an Access RTPCR System kit, and the primers that were used are as follows: inducible nitric oxide synthase (iNOS) forward $5^{\prime}$-CAA CAT CAG GTC GGC CAT CAC T-3', iNOS reverse 5'-ACC AGA GGC AGC ACA TCA AAG C-3 ${ }^{\prime}$; IL-6 forward 5'-ACA ACC ACG GCC TTC CTA C-3' ${ }^{\prime}$, IL-6 reverse 5' ${ }^{\prime}$ TCT CAT TTC CAC GAT TTC CCA G-3' ; TNF$\alpha$ forward $5^{\prime}$-CGA GTG ACA AGC CTG TAG CCC G-3', TNF$\alpha$ reverse $5^{\prime}$-GTC TTT GAG ATC CAT GCC GTT G- $3^{\prime} ; \beta$-actin forward $5^{\prime}$-TGC TGT CCC TGT ATG CCT CT- $3^{\prime}, \beta$-actin reverse $5^{\prime}$ TTT GAT GTC ACG CAC GAT TT-3'. $\beta$-actin was used as an internal control, and the results are expressed as the ratio of optimal density to $\beta$-actin.

\section{Results and discussion}

\subsection{Single-factor optimization for extraction}

In this study, the PAE method was applied to extract TQCPS from the stems of $T$. quadrispinosa. The extraction time, waterto-material ratio, extraction temperature, and extraction pressure were chosen for investigation, and the results are shown in Fig. 1.

3.1.1. Effect of extraction time on the yield of TQCPS. The effects of extraction time on the yield of TQCPS were studied from 10 to $50 \mathrm{~min}$ with other extraction conditions fixed as follows: water-to-material ratio of $30 \mathrm{~mL} \mathrm{~g}^{-1}$, extraction temperature at $50{ }^{\circ} \mathrm{C}$, and extraction pressure at $1.8 \mathrm{Mpa}$. As shown in Fig. 1a, the yield of TQCPS significantly increased from $1.83 \%$ to $3.62 \%$ when the extraction time changed from 10 to $30 \mathrm{~min}$, and then decreased as the extraction proceeded. This phenomenon could be explained by the long extraction time, which allowed the power induced by pressure to disrupt cells and increase the release rate of polysaccharide. ${ }^{22,35}$ However, under high pressure, an extraction time that is too long could 



Fig. 1 Effects of different extraction parameters on the yield of TQCPS: (a) extraction time (min); (b) extraction temperature ( $\left.{ }^{\circ} \mathrm{C}\right)$; (c) water-tomaterial ratio $\left(\mathrm{mL} \mathrm{g}^{-1}\right)$, and (d) extraction pressure $(\mathrm{Mpa})$.

result in the decrease in extraction yield because of the degradation of polysaccharides. ${ }^{36}$ Therefore, 30 min was considered to be the optimal extraction time.

3.1.2. Effect of extraction temperature on the yield of TQCPS. The effect of different temperatures on extraction yield of TQCPS was explored from 30 to $70{ }^{\circ} \mathrm{C}$ with other extraction conditions fixed as follows: extraction time of $30 \mathrm{~min}$, water-tomaterial ratio of $30 \mathrm{~mL} \mathrm{~g}^{-1}$, and extraction pressure at $1.8 \mathrm{Mpa}$. The results in Fig. $1 \mathrm{~b}$ show that the extraction temperature displayed a positive linear effect on the yield when the extraction temperature increased from 30 to $50{ }^{\circ} \mathrm{C}$. A maximum yield of $3.65 \%$ was observed at $50^{\circ} \mathrm{C}$, and after this point, the yield began to decrease. The increasing extraction temperature enhanced the polysaccharide solubility, diffusion coefficient, and increase in extraction yield. However, excessive extraction temperature could also lead to the hydrolyzation or degradation of polysaccharides. ${ }^{21}$ Hence, $50{ }^{\circ} \mathrm{C}$ was suitable for the extraction of TQCPS.

3.1.3. Effect of the water-to-material ratio on the yield of TQCPS. At a fixed extraction time of $30 \mathrm{~min}$, extraction temperature at $50^{\circ} \mathrm{C}$, and extraction pressure at $1.8 \mathrm{Mpa}$, the influences of the ratio of water to material on the yield of TQCPS are shown in Fig. 1c. The yield increased with increasing water-to-material ratio and reached a maximum of $3.64 \%$ at $30 \mathrm{~mL} \mathrm{~g}^{-1}$, while below and above $30 \mathrm{~mL} \mathrm{~g}^{-1}$, the yield began to slowly decrease. However, the effect of the water-to-material ratio on the yield was not significant $(P>$ $0.05)$. Therefore, this factor was fixed at $30 \mathrm{~mL} \mathrm{~g}^{-1}$ and ignored in further experiments.
3.1.4. Effect of extraction pressure on the yield of TQCPS. To study the effect of extraction pressure on the yield of TQCPS, the extraction process was carried out under different extraction pressures from 1.2 to $2.1 \mathrm{Mpa}$, while other extraction conditions were fixed as follows: extraction time of $30 \mathrm{~min}$, extraction temperature at $50{ }^{\circ} \mathrm{C}$, and waterto-material ratio of $30 \mathrm{~mL} \mathrm{~g}^{-1}$. As shown in Fig. 1d, the extraction yield remarkably increased with extraction pressure from 1.2 to $1.8 \mathrm{Mpa}$ and then decreased when the extraction pressure increased beyond $1.8 \mathrm{Mpa}$. With increasing pressure, the interaction between solvent and matrix and the solvent strength was increased. This resulted in an increase in the extraction yield. However, higher pressure disrupted the structure of polysaccharides, which led to a decline in extraction yield. ${ }^{37}$ Therefore, an extraction pressure of $1.8 \mathrm{Mpa}$ was preferred in the present experiment.

According to the single factor results, extraction time, extraction temperature, and extraction pressure more significantly affected the yield of TQCPS. Therefore, these three parameters were preferred for further experimentation.

\subsection{Optimization of TQCPS extraction by BBD}

3.2.1. Fitting the models. The influence of extraction time $\left(X_{1}\right)$, extraction temperature $\left(X_{2}\right)$, and extraction pressure $\left(X_{3}\right)$ on the extraction yield of TQCPS was evaluated by the BBD and consisted of three factors and three levels. The process variables 
Table 1 Box-Behnken design with independent variables and response values

\begin{tabular}{lrrrlll}
\hline & \multicolumn{3}{l}{ Independent variables } & & Response \\
\cline { 2 - 3 } Run & $X_{1}(\mathrm{~min})$ & $X_{2}\left({ }^{\circ} \mathrm{C}\right)$ & $X_{3}(\mathrm{Mpa})$ & & Experimental & Predicted \\
\hline 1 & $-1(20)$ & $-1(40)$ & $0(1.8)$ & & 2.50 & 2.55 \\
2 & $-1(20)$ & $1(60)$ & $0(1.8)$ & & 2.76 & 2.79 \\
3 & $-1(20)$ & $0(50)$ & $-1(1.5)$ & & 2.54 & 2.52 \\
4 & $-1(20)$ & $0(50)$ & $1(2.1)$ & & 3.20 & 3.22 \\
5 & $1(40)$ & $-1(40)$ & $0(1.8)$ & 3.36 & 3.33 \\
6 & $1(40)$ & $1(60)$ & $0(1.8)$ & 2.42 & 2.38 \\
7 & $1(40)$ & $0(50)$ & $-1(1.5)$ & 2.92 & 2.98 \\
8 & $1(40)$ & $0(50)$ & $1(2.1)$ & 3.02 & 3.04 \\
9 & $0(30)$ & $-1(40)$ & $-1(1.5)$ & 2.73 & 2.70 \\
10 & $0(30)$ & $-1(40)$ & $1(2.1)$ & 3.20 & 3.14 \\
11 & $0(30)$ & $1(60)$ & $-1(1.5)$ & 2.55 & 2.53 \\
12 & $0(30)$ & $1(60)$ & $1(2.1)$ & 2.66 & 2.69 \\
13 & $0(30)$ & $0(50)$ & $0(1.8)$ & 3.62 & 3.63 \\
14 & $0(30)$ & $0(50)$ & $0(1.8)$ & 3.68 & 3.63 \\
15 & $0(30)$ & $0(50)$ & $0(1.8)$ & 3.60 & 3.63 \\
& & & & &
\end{tabular}

and corresponding results are presented in Table 1. The yield of TQCPS ranged from $2.42 \%$ to $3.68 \%$. Experiment 14 (extraction time of $30 \mathrm{~min}$, extraction temperature at $50{ }^{\circ} \mathrm{C}$, and extraction pressure at $1.8 \mathrm{Mpa}$ ) represented the highest yield, while experiment 6 (extraction time of $40 \mathrm{~min}$, extraction temperature at $60{ }^{\circ} \mathrm{C}$, and extraction pressure at $1.8 \mathrm{Mpa}$ ) produced the lowest yield.

Based on multiple regression analysis of the experimental data, the model is expressed by a second-order polynomial equation as follows:

$$
\begin{aligned}
Y_{\text {yield }} & =3.63+0.09 X_{1}-0.18 X_{2}+0.17 X_{3}-0.30 X_{1} X_{2}-0.14 X_{1} X_{3} \\
& -0.09 X_{2} X_{3}-0.37 X_{1}^{2}-0.50 X_{2}^{2}-0.34 X_{3}^{2}
\end{aligned}
$$

The analysis of variance (ANOVA) for the second-order polynomial model response is given in Table 2. According to the results, the high $F$ value $(71.63)$ and the low $P$ value $(P<$
0.0001) suggested that the regression models are more significant. The goodness-of-fit of regression model was evaluated by determination coefficient $\left(R^{2}\right)$ and adjusted determination coefficient $\left(R_{\mathrm{adj}}^{2}\right)$. The high values of $R^{2}(0.9923)$ and $R_{\mathrm{adj}}^{2}$ (0.9785) indicated a satisfactory correlation between the experimental and predicted values. ${ }^{38}$ Furthermore, the low value of coefficient of variation (C.V.) at $2.15 \%$ and high value of adeq. precision at 24.059 demonstrated a very high degree of precision and a good deal of reliability of the experimental values. ${ }^{39}$ The low $F$ value (3.28) and the high $P$ value $(P=0.2425>0.05)$ for lack of fit suggested that it was not significant relative to the pure error, and a $24.25 \%$ chance could occur due to noise.

Usually, the $P$ value and $F$ value are used as a tool to check the significance of each coefficient. ${ }^{40}$ The smaller the $P$ value and the larger the $F$ value, the more significant the corresponding coefficient. According to Table 2, the yield of TQCPS was significantly influenced by the linear terms of extraction time $\left(X_{1}, P<0.05\right)$, extraction temperature $\left(X_{2}, P<0.001\right)$, extraction pressure $\left(X_{3}, P<0.001\right)$, and all three quadratic terms of $X_{1}{ }^{2}, X_{2}^{2}$, and $X_{3}^{2}(P<0.001)$. Among the interaction terms, $X_{1} X_{1}, X_{1} X_{3}$, and $X_{2} X_{3}$ were significant at $P<0.001,0.01$, and 0.05 levels, respectively. The $F$ value showed that the sequence of factors influencing the extraction yield was extraction temperature $>$ extraction pressure $>$ extraction time, and the order of interaction effect was $X_{1} X_{2}>X_{1} X_{3}>X_{2} X_{3}$.

3.2.2. Response surface analysis of extraction yield of TQCPS. The three-dimensional (3D) response surface plots and two-dimensional (2D) contour plots have been proved to be effective methods to illustrate the correlations between variables and response as well as the influence of interactions between different variables. ${ }^{41}$ The steepness of the $3 \mathrm{D}$ response surface demonstrates the different influence of variables on the response. The steeper the $3 \mathrm{D}$ response surface, the more significant the influence of interaction between the variable and the response. The different shapes of the $2 \mathrm{D}$ contour plots indicate the different interactions between the two corresponding variables. Elliptical contours imply that the interaction of corresponding variables was significant, while the

\begin{tabular}{|c|c|c|c|c|c|c|c|}
\hline Model & 3.63 & 2.65 & 9 & 0.29 & 71.63 & $<0.0001$ & $* * *<0.001$ \\
\hline$X_{1}$ & 0.09 & 0.065 & 1 & 0.065 & 15.79 & 0.0106 & $*<0.05$ \\
\hline$X_{3}$ & 0.17 & 0.22 & 1 & 0.22 & 54.70 & 0.0007 & $* * *<0.001$ \\
\hline$X_{1} X_{2}$ & -0.30 & 0.36 & 1 & 0.36 & 87.73 & 0.0002 & $* * *<0.001$ \\
\hline$X_{1} X_{3}$ & -0.14 & 0.078 & 1 & 0.078 & 19.11 & 0.0072 & $* *<0.01$ \\
\hline$X_{2}^{2}$ & -0.50 & 0.94 & 1 & 0.94 & 228.72 & $<0.0001$ & $* * *<0.001$ \\
\hline$X_{3}^{2}$ & -0.34 & 0.44 & 1 & 0.44 & 106.59 & 0.0001 & $* * *<0.001$ \\
\hline \multicolumn{2}{|l|}{ Residual } & 0.021 & 5 & $4.10 \times 10-3$ & & & \\
\hline \multicolumn{2}{|l|}{ Lack of fit } & 0.017 & 3 & $5.68 \times 10^{-3}$ & 3.28 & 0.2424 & $>0.05$ \\
\hline \multicolumn{2}{|l|}{ Pure error } & $3.47 \times 10^{-3}$ & 2 & $1.73 \times 10^{-3}$ & & & \\
\hline \multicolumn{2}{|l|}{ Col total } & 2.67 & 14 & & & & \\
\hline
\end{tabular}

Table 2 Analysis of variables for regression model of response under extraction conditions ${ }^{a}$ 
circular contours indicate that the interaction of corresponding variables was negligible. ${ }^{42}$

In the present study, the $3 \mathrm{D}$ response surface and $2 \mathrm{D}$ contour plots illustrate the relationship between extraction time $\left(X_{1}\right)$, extraction temperature $\left(X_{2}\right)$, extraction pressure $\left(X_{3}\right)$, and extraction yield of TQCPS (Fig. 2). Fig. 2a and b illustrate the effect of extraction time and extraction temperature on the yield when fixing the extraction pressure at the zero level (1.8 Mpa).
The extraction temperature exhibited a quadratic effect on the yield. When the extraction time was maintained at a lower level, the yield initially increased and then decreased with increasing extraction temperature. At the same time, the result indicated that the curved surface of the extraction temperature was steeper than that of extraction time. This signified that the influences of extraction temperature on the extraction yield were more significant than that of extraction time.
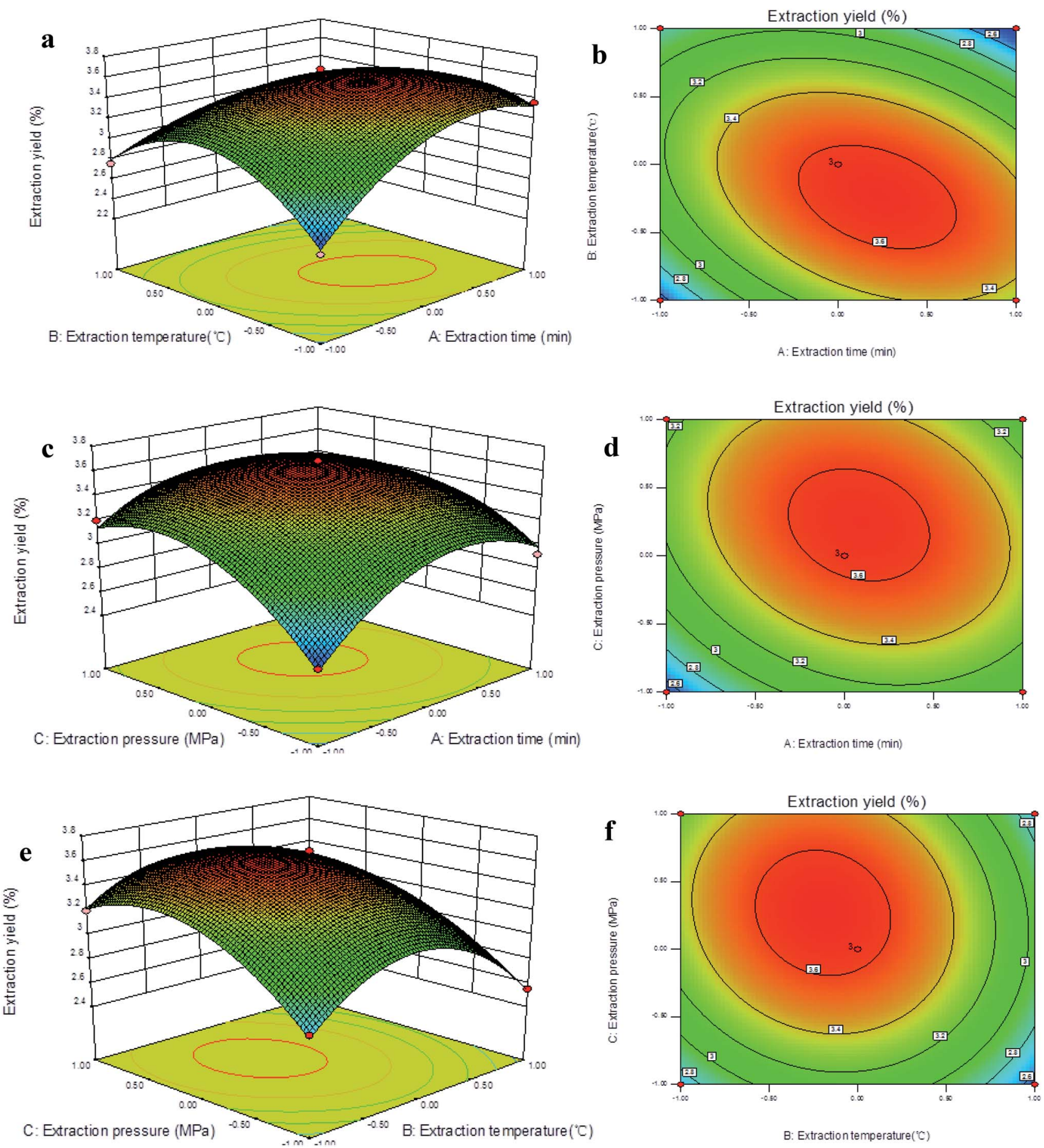

Fig. 2 Response surface plots ( $a, c$ and e) and contour plots ( $b, d$ and f) for the interactive effects of extraction time, extraction temperature, and extraction pressure on the yield of polysaccharides. 




Fig. 3 Scanning electron microscopy images of T. quadrispinosa stem samples: (a) untreated raw material and (b) material treated by PAE under optimum conditions.

Furthermore, the yield was significantly influenced by the interaction between extraction temperature and extraction time because of the most elliptical contour shape among all of them.

Fig. $2 c$ and d exhibit the effects of extraction time and extraction pressure on the yield when fixing the extraction temperature at the zero level $\left(50^{\circ} \mathrm{C}\right)$, and show that both the extraction time and extraction pressure had a positive impact on the yield. There was an obvious increase in the yield when increasing extraction time and extraction pressure, and the yield slightly decreased when the extraction time and pressure increased up to a threshold level. Additionally, the extraction time and pressure exhibited the lowest interaction effects because of the smallest degree of an elliptical shape on 2D contour plots. Fig. 2e and f show the mutual interactions between extraction temperature and extraction pressure on the yield of TQCPS when fixing the extraction time at $30 \mathrm{~min}$. When the extraction temperature was maintained at a lower level, the yield increased with increasing extraction pressure. However, the yield increased and then slightly decreased with increasing extraction temperature when the extraction pressure was fixed. It was indicated that a greater yield could be obtained when a higher extraction pressure and a lower extraction temperature was selected, and the TQCPS was more likely to be degraded under the condition of high temperature.

3.2.3. Verification of models and comparison with the classical method. The object of this study was to optimize the PAE conditions for maximum extraction yield of TQCPS. The optimum extraction conditions calculated from the models are as follows: extraction time of $31.8 \mathrm{~min}$, extraction temperature at $47.5{ }^{\circ} \mathrm{C}$, and extraction pressure at $1.872 \mathrm{Mpa}$, with the highest predicted yield of polysaccharides of $3.683 \%$. In order to easily control the extraction parameters during actual extraction experiments, the optimal extraction conditions were carried out with the slight modifications of extraction time of $32 \mathrm{~min}$, extraction temperature at $47^{\circ} \mathrm{C}$, and extraction pressure at 1.87 Mpa. The mean yield of $3.72 \pm 0.13 \%(n=3)$ was obtained under these conditions, which was in agreement with the predicted values, suggesting the suitability of the fitted response surface model. In this study, the effect of PAE was compared with that of HWE. At the extraction time of $240 \mathrm{~min}$ and extraction temperature at $90^{\circ} \mathrm{C}$ for HWE, the TQCPS yield was $2.32 \pm 0.21 \%(n=3)$. Thus, the PAE method remarkably enhanced the extraction yield, with further advantages of short extraction time and low extraction temperature. These results indicated that the PAE method is a promising environmentally friendly technique that offers greater efficiency than HWE.

\subsection{Analysis of microscopic changes}

To study the structural alteration of samples treated by PAE, the T. quadrispinosa stem samples were observed by SEM. As shown in Fig. 3a, it was obvious that there was a complete parenchyma and no destruction of cell walls for the untreated samples. However, samples subjected to PAE exhibited a lack of cellular integrity, and there were many huge perforations on the


Fig. 4 (a) UV-vis and (b) FT-IR spectra of TQCPS. 

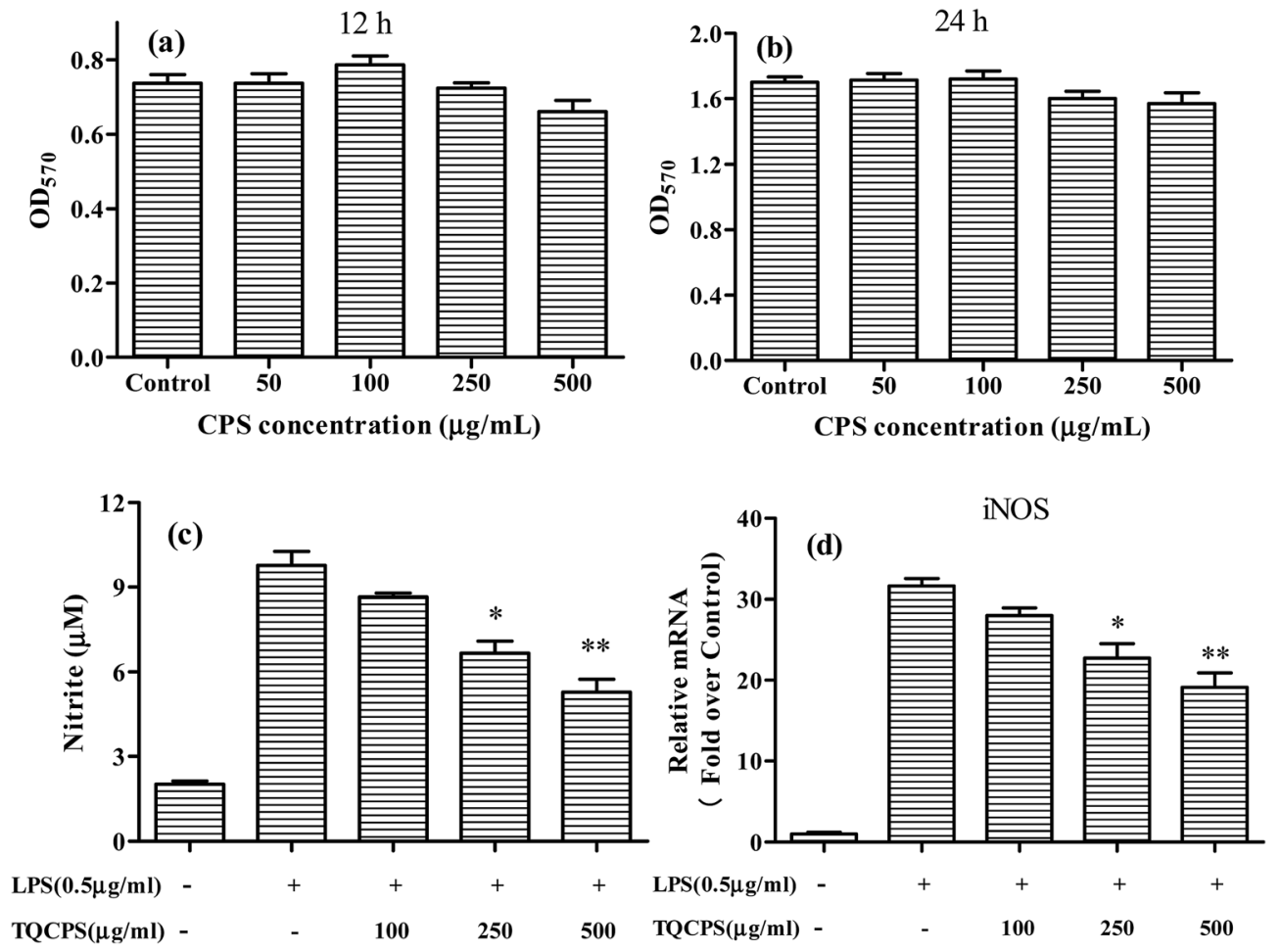

Fig. 5 Effects of TQCPS on (a and b) proliferation of RAW 264.7 cells, (c) LPS-induced NO generation, and (d) iNOS mRNA expression. (c) RAW 264.7 cells were treated with LPS with or without different concentrations of TQCPS for $24 \mathrm{~h}$. The levels of NO were detected by Griess reaction. (d) RAW 264.7 cells were treated with LPS with or without different concentrations of TQCPS for $12 \mathrm{~h}$. The mRNA level of iNOS was determined by RT-PCR analysis. LPS $\left(0.5 \mathrm{~mL}^{-1}\right)$ was used as a positive control. The data are presented as the mean \pm SD $(n=3)$. *p<0.05,**p<0.01 compared with control groups.

external surfaces (Fig. 3b). The damaged cells easily released their intracellular polysaccharides into the extraction solvent, which contributed to the higher extraction efficiency. ${ }^{43}$

\subsection{Preliminary characterization of TQCPS}

The chemical composition of TQCPS was determined, and the amounts of carbohydrate, uronic acid, protein, and total polyphenols in TQCPS were $62.42 \%, 17.84 \%, 2.44 \%$, and $0.53 \%$, respectively. The UV-vis spectrum of TQCPS was scanned at the range of $200-600 \mathrm{~nm}$. As shown in Fig. 4a, there was weak absorbance at $280 \mathrm{~nm}$, indicating the presence of proteins, which was consistent with the results of the chemical composition analysis of TQCPS. Additionally, there was no absorbance at 260 or $520 \mathrm{~nm}$ in the UV spectrum, indicating the absence of nucleic acid and pigment, respectively. The FT-IR spectrum of TQCPS was recorded in the range of $4000-400 \mathrm{~cm}^{-1}$ (Fig. 4b). A strong absorbance band at $3416 \mathrm{~cm}^{-1}$ represents $\mathrm{O}-\mathrm{H}$ stretching vibration in the constituent sugar residues. The weak band at $2920 \mathrm{~cm}^{-1}$ indicates $\mathrm{C}-\mathrm{H}$ stretching vibration in the sugar ring. ${ }^{44}$ The bands at 1748 and $1614 \mathrm{~cm}^{-1}$ denote the unique absorption of $\mathrm{C}=\mathrm{O}$ and $\mathrm{COO}^{-}$belonging to uranic acid. ${ }^{45}$ The bands at 1448,1324 , and $1230 \mathrm{~cm}^{-1}$ were assigned to $\mathrm{C}-\mathrm{O}$ stretching vibrations and $\mathrm{O}-\mathrm{H}$ deformation vibrations. ${ }^{46}$ The strong absorption bands in the $1060 \mathrm{~cm}^{-1}$ region (1108, $1016 \mathrm{~cm}^{-1}$ ) were identified to be the vibrations of the C-O-C bond. ${ }^{47}$ Furthermore, the bands at 962 and $836 \mathrm{~cm}^{-1}$ relating to $\alpha$ - and $\beta$-configurations were also characteristic of glycosidic structures. ${ }^{48}$

\subsection{Anti-inflammatory activity of TQCPS}

LPS is a prototypical bacterial endotoxin that can initiate a variety of inflammatory responses by directly activating macrophages. The activated macrophages produce increasing amounts of NO and cytokines such as IL- 1 , IL- 6 , and TNF- $\alpha$, all of which are involved in the pathogenesis of many diseases. Therefore, macrophages in the presence of LPS have been widely used for evaluating the anti-inflammatory potential of samples in vitro. ${ }^{49}$ In this study, RAW 264.7 , the most commonly used mouse macrophage cell line, was selected to evaluate the anti-inflammatory activity of TQCPS.

3.5.1. Cytotoxicity of TQCPS on RAW 264.7 cells. Initially, to evaluate the anti-inflammatory activity of TQCPS, its possible cytotoxic effect on RAW 264.7 cells was tested by the MTT method. The cell viability was performed at $50-500 \mu \mathrm{g} \mathrm{mL}$ concentration of TQCPS for 12 and $24 \mathrm{~h}$. The results in Fig. $5 \mathrm{a}$ and $\mathrm{b}$ show that at all tested concentrations, the viability of RAW 264.7 cells was not significantly influenced by TQCPS treatment, which indicates that the concentrations of TQCPS used in this study are considered to be non-cytotoxic.

3.5.2. Suppressive effect of TQCPS on NO secretion and iNOS expression in LPS-induced RAW 264.7 cells. It was reported that NO plays an important role in inflammatory 
responses, and the level of NO secretion has been correlated with the gene expression level of iNOS. ${ }^{50}$ In this study, the effects of TQCPS on NO production and gene expression in RAW 264.7 macrophage cells were examined using the Griess assay and RT-PCR analysis, respectively.

The results are shown in Fig. 5c and d. As shown in Fig. 5c, LPS alone markedly increased NO production up to $9.77 \mu \mathrm{M}$ compared to $2.01 \mu \mathrm{M}$ NO from untreated RAW 264.7 cells. The level of NO decreased in a dose-dependent manner when RAW 264.7 cells were co-incubated with TQCPS and LPS. When TQCPS was added at $500 \mu \mathrm{g} \mathrm{mL} \mathrm{m}^{-1}$, the production level of NO was determined to be $5.28 \mu \mathrm{M}$, a significant $46 \%$ decrease compared to cells treated with LPS alone $(p<0.01)$. Correspondingly, the stimulation of RAW 264.7 cells with LPS was noteworthy and increased mRNA expression of iNOS against untreated cells by 31.6 fold. However, the mRNA level of iNOS decreased from 28.0 fold to 19.1 fold after treatment with TQCPS at concentrations ranging from 100 to $500 \mu \mathrm{g} \mathrm{mL} \mathrm{m}^{-1}$ in a dose-dependent manner (Fig. 5d). These results suggest that TQCPS could decrease NO production by the downregulation of iNOS expression at a transcriptional level in RAW 264.7 cells stimulated by LPS.

3.5.3. Suppressive effect of TQCPS on cytokine secretion and mRNA expression in LPS-induced RAW 264.7 cells. Activated macrophages generate and sequentially secrete a variety of pro-inflammatory cytokines such as TNF- $\alpha$, IL-1, and IL-6, which have been known to play important roles in proinflammatory responses. ${ }^{51}$ In an attempt to elucidate how TQCPS acts as an anti-inflammatory substance, the effects of TQCPS on inflammatory cytokine secretion and gene expression in LPS 264.7 macrophage cells were examined using ELISA assays and RT-PCR analysis, respectively.

The results revealed that the secretion of TNF- $\alpha$ and IL-6 significantly increased from 43.6 and $4.7 \mathrm{pg} \mathrm{mL}^{-1}$ to 634.3 and $197.7 \mathrm{pg} \mathrm{mL}^{-1}$, respectively, when cells were stimulated by LPS. The secretion levels of TNF- $\alpha$ and IL- 6 were decreased to 331.3 and $108.7 \mathrm{pg} \mathrm{mL} \mathrm{m}^{-1}$, respectively, when $500 \mu \mathrm{g} \mathrm{mL} \mathrm{m}^{-1}$ TQCPS was added to the LPS-stimulated RAW 264.7 cells (Fig. 6a and b). Furthermore, the effect of TQCPS on the level of mRNA expression was also determined by RT-PCR. As shown in Fig. $6 \mathrm{c}$ and $\mathrm{d}$, there was a dose-dependent decrease in the mRNA expression of TNF- $\alpha$ and IL-6 (20.57 and 14.18 fold, respectively, at $500 \mu \mathrm{g} \mathrm{mL} \mathrm{m}^{-1}$ TQCPS) compared to the stimulation group (31.38 and 23.44 fold, respectively). These results strongly indicate that TQCPS could suppress the expression and secretion of inflammatory cytokines in LPS-induced RAW 264.7 cells.

It is well known that the bioactivities of polysaccharides are mostly dependent on their physicochemical characteristics including composition, distribution of average molecular weight, monosaccharide component, backbone, connection location, repeating unit, and advanced structures. ${ }^{52,53}$ Thus, it is necessary to carry out a further study on the purification and


Fig. 6 Effects of TQCPS on LPS-induced IL- 6 and TNF- $\alpha$ secretion, and mRNA expression. ( $a$ and b) RAW 264.7 cells were treated with LPS with or without different concentrations of TQCPS for $24 \mathrm{~h}$. The levels of IL- 6 and TNF- $\alpha$ were analyzed by ELISA. (c and d) RAW 264.7 cells were treated with LPS with or without different concentrations of TQCPS for $12 \mathrm{~h}$. The mRNA levels of IL- 6 and TNF- $\alpha$ were determined by RT-PCR analysis. LPS $\left(0.5 \mu \mathrm{g} \mathrm{mL}^{-1}\right)$ was used as a positive control. The data are presented as the mean $\pm \mathrm{SD}(n=3)$. ${ }^{*} p<0.05, * * p<0.01 \mathrm{compared} \mathrm{with}$ the control group. 
physical characterization analysis of purified polysaccharide and its detailed mechanism of anti-inflammatory activity.

\section{Conclusions}

In this study, pressurized-assisted extraction was successfully used to extract polysaccharides from the stem of T. quadrispinosa. Response surface methodology was employed to optimize extraction conditions. Fixing the ratio of water to material at $30 \mathrm{~mL} \mathrm{~g}^{-1}$, the optimal extraction conditions were as follows: extraction time of $32 \mathrm{~min}$, extraction temperature at $47{ }^{\circ} \mathrm{C}$, and extraction pressure at $1.87 \mathrm{Mpa}$, with the highest yield of polysaccharides at $3.72 \% \pm 0.13 \%$. The application of pressurized-assisted extraction significantly decreased extraction time, and improved the extraction efficiency compared with the conventional hot water extraction method. Investigation of the anti-inflammatory activity of TQCPS on murine macrophage cell line RAW 264.7 revealed that TQCPS significantly downregulates the production of $\mathrm{NO}$ and the secretion of inflammatory cytokines, including IL-6 and TNF- $\alpha$. This indicates that TQCPS can be explored as a potential antiinflammatory agent for use in medicine.

\section{Conflicts of interest}

The authors declare that they have no competing interests.

\section{Acknowledgements}

The financial support for this investigation was given by the National Natural Science Foundation of China (81402840), Natural Science Foundation of Jiangsu Province, China (BK20130495), College of Natural Science Research Program of Jiangsu Province, China (13KJB350001), and the Natural Science Foundation of Jiangsu Province, Administration of Traditional Chinese Medicine, China (YB2017101).

\section{References}

1 B. T. Zhao, J. Liu, X. Chen, J. Zhang and J. L. Wang, RSC Adv., 2018, 8, 11731-11743.

2 I. Ghazala, A. Sila, F. Frikha, D. Driss, S. Ellouz-Chaabouni and A. Haddar, J. Food Sci. Technol., 2015, 52, 6953-6965.

3 C. L. Lu, W. Zhu, M. Wang, M. M. Hu, W. L. Chen, X. J. Xu and C. J. Lu, Carbohydr. Polym., 2015, 122, 428-436.

4 Q. Y. Wu, H. S. Qu, J. Q. Jia, C. Kuang, Y. Wen, H. Yan and Z. Z. Gui, Carbohydr. Polym., 2015, 132, 31-40.

5 C. Chen, L. J. You, A. M. Abbasi, X. Fu and R. H. Liu, Carbohydr. Polym., 2015, 130, 122-132.

6 C. H. Liao and J. Y. Lin, Food Chem., 2012, 135, 1818-1827.

7 H. L. Liu, R. Xu, L. L. Feng, W. J. Guo, N. Cao, C. Qian, P. Teng, L. Wang, X. F. Wu, Y. Sun, J. X. Li, Y. Shen and Q. Xu, PLoS One, 2012, 7, 37168.

8 T. Luo, J. Qin, M. Liu, J. Luo, F. Ding, M. L. Wang and L. M. Zheng, Inflammation Res., 2015, 64, 205-212.
9 Y. Diao, Y. Q. Xin, Y. Zhou, N. Li, X. L. Pan, S. M. Qi, Z. L. Qi, Y. M. Xu, L. Luo, H. G. Wan, L. Lan and Z. M. Yin, Int. Immunopharmacol., 2014, 18, 12-19.

10 J. J. Cheng, C. H. Chao, P. C. Chang and M. K. Lu, Food Hydrocolloids, 2016, 53, 37-45.

11 R. Z. Chen, S. Z. Li, C. M. Liu, S. M. Yang and X. L. Li, Process Biochem., 2012, 47, 2040-2050.

12 J. B. Pu, B. H. Xia, Y. J. Hu, H. J. Zhang, J. Chen, J. Zhou, W. Q. Liang and P. Xu, Molecules, 2015, 20, 22220-22235.

13 C. L. Cao, Q. Huang, B. Zhang, C. Li and X. Fu, Int. J. Biol. Macromol., 2018, 109, 357-368.

14 S. Q. Qian, X. H. Fang, D. M. Dan, E. J. Diao and Z. X. Lu, RSC $A d v .$, 2018, 8, 42145-42152.

15 Y. Q. Xu, F. Cai, Z. Y. Yu, L. Zhang, X. G. Li, Y. Yang and G. J. Liu, Food Chem., 2016, 194, 650-658.

16 M. Plaza and C. Turner, TrAC, Trends Anal. Chem., 2015, 71, 39-54.

17 H. N. Zhang, W. Tchabo and Y. K. Ma, Emir. J. Food Agric., 2017, 29, 815-819.

18 H. Kamali, T. A. Sani, A. Mohammadi, P. Alesheikh, E. Khodaverdi and F. Hadizadeh, J. Supercrit. Fluids, 2018, 133, 535-541.

19 W. Tchabo, Y. K. Ma, E. Kwaw, H. N. Zhang, X. Li and N. A. Afoakwah, Food Bioprocess Technol., 2017, 10, 12101223.

20 F. J. Leyva-Jimenez, J. Lozano-Sanchez, I. Borras-Linares, D. Arraez-Roman and A. Segura-Carretero, Food Res. Int., 2018, 109, 213-222.

21 Q. Yuan, S. Lin, Y. Fu, X. R. Nie, W. Liu, Y. Su, Q. H. Han, L. Zhao, Q. Zhang, D. R. Lin, W. Qin and D. T. Wu, Int. J. Biol. Macromol., 2019, 127, 178-186.

22 J. Gao, L. Z. Lin, B. G. Sun and M. M. Zhao, Food Funct., 2017, 8, 3043-3052.

$23 \mathrm{H}$. Kamali, E. Khodaverdi, F. Hadizadeh and S. H. Ghaziaskar, J. Supercrit. Fluids, 2016, 107, 307-314.

24 H. N. Zhang and Y. K. Ma, Czech J. Food Sci., 2017, 35, 180187.

25 M. L. Zhong, S. Y. Huang, H. H. Wang, Y. L. Huang, J. R. Xu and L. J. Zhang, RSC Adv., 2019, 9, 1576-1585.

26 X. L. Luo, J. M. Cui, H. H. Zhang, Y. Q. Duan, D. Zhang, M. H. Cai and G. Y. Chen, Ind. Crops Prod., 2018, 112, 296304.

27 P. Y. Chiang and J. Y. Ciou, LWT-Food Sci. Technol., 2010, 43, 361-365.

28 H. N. Yu and S. R. Shen, LWT-Food Sci. Technol., 2015, 61, 238-243.

29 A. Raza, F. Li, X. Q. Xu and J. Tang, Int. J. Biol. Macromol., 2017, 94, 335-344.

30 J. Fang, T. Seki and H. Maeda, Adv. Drug Delivery Rev., 2009, 61, 290-302.

31 M. Dubois, K. A. Gilles, J. K. Hamilton, P. Rebers and F. Smith, Anal. Chem., 1956, 28, 350-356.

32 N. Blumenkrantz and G. Asboe-Hansen, Anal. Biochem., 1973, 54, 484-489.

33 M. M. Bradford, Anal. Biochem., 1976, 72, 248-254. 
34 M. F. Vázquez, L. R. Comini, J. M. Milanesio, S. N. Montoya, J. L. Cabrera, S. Bottini and R. E. Martini, J. Supercrit. Fluids, 2015, 101, 170-175.

35 A. Valdés, L. Vidal, A. Beltrán, A. Canals and M. C. Garrigós, J. Agric. Food Chem., 2015, 63, 5395-5402.

36 Ó. Benito-Román, E. Alonso and M. Cocero, J. Supercrit. Fluids, 2013, 73, 120-125.

37 J. L. Liu, S. L. Zheng, Q. J. Fan, J. C. Yuan, S. M. Yang and F. L. Kong, Int. J. Biol. Macromol., 2015, 76, 80-85.

38 X. J. Hou and W. Chen, Carbohydr. Polym., 2008, 72, 67-74.

39 X. F. Wang, H. Zhang, Z. Y. Wang and H. N. Bai, RSC Adv., 2015, 5, 106800-106808.

40 C. P. Zhu and X. L. Liu, Carbohydr. Polym., 2013, 92, 11971202.

41 S. A. Shen, D. J. Chen, X. Li, T. Li, M. Yuan, Y. H. Zhou and C. B. Ding, Carbohydr. Polym., 2014, 104, 80-86.

42 J. Zhang, S. Y. Jia, Y. Liu, S. H. Wu and J. Y. Ran, Carbohydr. Polym., 2011, 86, 1089-1092.

43 J. Xi and L. G. Yan, Sep. Purif. Technol., 2017, 175, 170-176. 44 C. X. Yang, N. He, X. P. Ling, M. L. Ye, C. X. Zhang, W. Y. Shao, C. Y. Yao, Z. Y. Wang and Q. B. Li, Sep. Purif. Technol., 2008, 63, 226-230.
45 W. K. Hsu, T. H. Hsu, F. Y. Lin, Y. K. Cheng and J. P. W. Yang, Carbohydr. Polym., 2013, 92, 297-306.

46 X. Jin, Q. L. Wang, X. Yang, M. Guo, W. J. Li, J. X. Shi, M. AduFrimpong, X. M. Xu, W. W. Deng and J. N. Yu, Int. J. Food Sci. Technol., 2018, 53(10), 2298-2307.

47 X. J. Jia, C. Zhang, J. F. Qiu, L. L. Wang, J. L. Bao, K. Wang, Y. L. Zhang, M. W. Chen, J. B. Wan, H. X. Su, J. P. Han and C. W. He, Carbohydr. Polym., 2015, 132, 67-71.

48 Q. X. Yuan, L. Y. Zhao, Q. Q. Cha, Y. Sun, H. Ye and X. X. Zeng, J. Agric. Food Chem., 2015, 63, 7986-7994.

49 Y. Diao, Y. Q. Xin, Y. Zhou, N. Li, X. L. Pan, S. M. Qi, Z. L. Qi, Y. M. Xu, L. Luo, H. G. Wan, L. Lan and Z. M. Yin, Int. Immunopharmacol., 2014, 18, 12-19.

50 W. W. Lee, G. Ahn, J. P. W. Arachchillage, Y. M. Kim, S. K. Kim, B. J. Lee and Y. J. Jeon, J. Med. Food, 2011, 14, 1546-1553.

51 T. T. Zhang, C. L. Lu, J. G. Jiang, M. Wang, D. M. Wang and W. Zhu, Carbohydr. Polym., 2015, 130, 307-315.

52 X. Y. Li and L. Wang, Int. J. Biol. Macromol., 2016, 83, 270276.

53 Z. F. Zhang, G. Y. Lv, H. J. Pan, L. G. Shi and L. F. Fan, Food Sci. Technol. Res., 2011, 17, 461-470. 